\title{
Combining Ability of wheat yield and Some yield Attributes Under Normal and Water Stress Conditions
}

\author{
EL-Banna, M.N. ${ }^{1}$, M.A. Gomaa ${ }^{1}$, M.A. Nassar ${ }^{1}$, H.A. Ashoush ${ }^{2}$ and Y.A. EL-Gohary ${ }^{2}$ \\ 1- Plant Production Dept., Faculty of Agriculture, Saba Basha, Alexandria University, Egypt. \\ 2- Wheat Research Dept., Field Crops Research Institute, Agricultural Research Center, Egypt.
}

ABSTRACT: A half diallel cross among six parents of wheat (Triticum aestivum L.) was achieved for combining ability under recommended irrigation and water stress at Etay EL-Baroud Agricultural Research Station, Agricultural Research Center, Egypt, during 2009/2010 and 2010/2011 seasons in RCBD with three replications. Data were recorded from $F_{1}$ generation for days to heading, days to physiological maturity, plant height, flag leaf area, spike length, number of spikelets spike ${ }^{-1}$, number of kernels spike ${ }^{-1}$, number of spikes plant ${ }^{-1}, 1000$-kernel weight and grain yield plant ${ }^{-1}$. Mean squares for genotypes, parents, crosses and parent vs. crosses were significant for all the studied traits in both irrigation treatments. General combining ability (GCA) and specific combining ability (SCA) mean squares were significant for all the studied traits in both irrigation treatments. High GCA/SCA ratios which largely exceeded the unity were detected for all studied traits, except for grain yieldplant ${ }^{-1}$ under the study in both conditions. Wheat genotype Line $1\left(\mathrm{P}_{6}\right)$ showed the maximum desirable GCA values for days to heading, days to physiological maturity, flag leaf area, spike length, under water stress, number of kernels spike ${ }^{-1}$ and 1000-kernel weight under both conditions. For shortness, the cultivar Sakha $93\left(P_{2}\right)$ proved to be the best general combiner for plant height under both environments. Meanwhile, Sham 6 under normal conditions and Sahel 1 under water stress conditions were the best combiners for plant height concerning breeding for tallness. The cultivar Gemmeiza 9 proved to be a good general combiner for number of spikelets spike ${ }^{-1}$ under both conditions. The cultivar Misr 1 found to be the best general combiner for number of spikes plant ${ }^{-1}$ and grain yield plant ${ }^{-1}$ under normal condition, while the cultivar Sakha 93 found to be the best general combiner for number of spikes plant $^{-1}$ and grain yield plant ${ }^{-1}$ under water stress condition. The cross (Sahel $1 \times$ Sham 6) showed the maximum desirable SCA values for days to heading and days to physiological maturity under both water regime treatments. The cross (Misr $1 \times$ Line 1) exhibited the maximum SCA for plant height under normal conditions, while (Sahel $1 \times$ Gemmeiza 9) showed the maximum SCA for plant height under water stress condition. The cross (Sahel $1 \times$ Misr 1) showed maximum SCA value for flag leaf area under both environments. The cross (Sham $6 \times$ Line 1) was the best specific combiners for spike length under normal conditions, while (Sahel $1 \times$ Sham 6 ) was the best for spike length under stress conditions. The best specific combiner for kernels spike $e^{-1}$ under both conditions was the cross (Misr $1 \mathrm{x}$ Line 1). The cross (Sakha $93 \times$ Misr 1) showed the best SCA for number of spikes plant ${ }^{-1}$ under normal conditions while the cross (Sakha $93 \times$ Line 1) was the best one under water stress conditions. For 1000-kernel weight the cross (Gemmeiza $9 \times$ Misr 1) was the best specific combiner under both irrigation conditions. The cross (Sakha $93 \times$ Line 1) exhibited the maximum SCA for grain yield plant ${ }^{-1}$ under normal conditions while the cross (Sakha $93 \times$ Gemmeiza 9) was the best one under water stress conditions. The crosses (Sakha $93 \times$ Gemmeiza 9), (Sakha $93 \times$ Line 1) and (Misr $1 \times$ Line 1) were prospective in wheat breeding programs since they expressed significant and desirable $\left(\hat{\mathrm{s}}_{\mathrm{i}}\right)$ effects for most traits.

Keywords: Bread wheat, General combining ability (GCA), Specific combining ability (SCA), Normal irrigation, Water stress.

\section{INTRODUCTION}

Wheat (Triticum aestivum L.) is the most important cereal crop in Egypt as well as in most countries over the world. Egypt's strategy is to minimize the food gap of this crop particular throughout vertical improvement and horizontal expansion. Although the cultivated area is restricted, enormous activities in new land reclamation 
and cultivation succeeded to add more land for food production. But, the limited water resources put limitations. Moreover, drought is a worldwide issue that impacts seriously on the security of food production and the global climate change makes this even worse (Elisabeth et al., 2009). Therefore, breeding and correct cultivation of cultivars having high yield potentialities are the main way to produce wheat crop to fill the gap between production and increased population.

Breeders should concentrate on development of productive wheat varieties by crossing good general combining lines for grain yield and selecting transgressive segregates from the resulting hybrids. Information regarding general and specific combining ability of wheat genotypes is a prerequisite to launch a successful wheat breeding program. Diallel mating design has been extensively used to analyze the combining ability effects of wheat genotypes and also to provide information regarding genetic mechanisms controlling grain yield and other traits.

Significant differences due to GCA and SCA were observed for the studied traits (Pang et al., 2010). Saeed et al. (2010) observed significant differences among genotypic mean in all of the traits under both conditions, they reported that; GCA and SCA differences were significant for all the traits under study except spike density and 100-grain weight in both conditions. Khan et al. (2007) found that, the additive gene effects were operating in plant height, biomass plant ${ }^{-1}$, number of grains spike and grain yield plant ${ }^{-1}$, while number of tillers plant ${ }^{-1}$ and 1000 -grain weight were controlled by non-additive gene effects. Golparvar (2013) found that GCA to SCA mean square ratio was significant for non of traits, indicating that non additive effects of genes were more important than additive effect for all studied traits.

The objectives of the present investigation were to assess the variations among wheat genotypes and available crosses for water stress tolerance characteristics, find out the good general combining genotypes for sound breeding program and to select high yielding combiners for the development of productive wheat varieties and mark crosses with better specific combining ability for yield related traits by employing diallel cross technique and to determine suitable measurements for drought resistance in wheat genotypes.

\section{MATERIALS AND METHODS}

The present investigation was carried out at Etay EL-Baroud Agricultural Research Station, Agricultural Research Center, Egypt, during the successive wheatgrowing seasons, 2009/2010 and 2010/2011. Six common wheat genotypes (Triticum aestivum $\mathrm{L}$. em Thell) representing a wide divergent were selected to establish the experimental materials for this study. The names, pedigree and origin of these varieties and/or lines are presented in Table (1). 
Table (1): Code number, names, pedigree and origin of six parents of bread wheat

\begin{tabular}{|c|c|c|c|}
\hline No. & Name & Pedigree & Origin \\
\hline P1 & Sahel 1 & $\begin{array}{l}\text { NS.732/PIMA/VEERY"S" } \\
\text { SD735-4SD-1SD-1SD-0SD }\end{array}$ & Egypt \\
\hline $\mathrm{P} 2$ & Sakha 93 & $\begin{array}{l}\text { Sakha } 92 \text { / TR } 810328 \\
\text { S8871-1S-2S-1S- 0S }\end{array}$ & Egypt \\
\hline P3 & Gemmeiza 9 & $\begin{array}{l}\text { Ald "S"/ Huac// Cmh74A .630/ Sx } \\
\text { CGM4583 -5GM-1GM- 0GM }\end{array}$ & Egypt \\
\hline $\mathrm{P} 4$ & Misr 1 & $\begin{array}{l}\text { OASIS/SKAUZ//4*BCN/3/2*PASTOR } \\
\text { CMSS00Y01881T-050M-030Y-030M- } \\
\text { 030WGY-33M-0Y-0S. }\end{array}$ & Egypt \\
\hline P5 & Sham 6 & $\begin{array}{l}\text { W-3918-A/JUPATECO-73 } \\
\text { CM-39992-8M-7Y-0M-0AP-0SYR }\end{array}$ & Syria \\
\hline P6 & Line\# 1 & $\begin{array}{l}\text { KAUZ/PASTOR } \\
\text { CMSSB0025S-48Y-010M-010Y-010M-9Y-0M }\end{array}$ & CIMMYT \\
\hline
\end{tabular}

In 2009/2010 season, grains for each of the parental genotypes were sown at various dates at 7 days interval for 4 weeks to provide synchronized flowering among the genotypes. All possible cross combinations excluding reciprocals were made among the six genotypes to produce their fifteen $F_{1}$ crosses. Hybridization was made by hand for enough number of spikes on main stems of each parent after 2-5 days from hand emasculation according to weather conditions.

In 2010/2011 wheat growing season, the six parents and their fifteen $F_{1}$ hybrid seeds were sown on $1^{\text {st }}$ of December in two experiments. The twenty one (21) entries were evaluated in two separate irrigation regime experiments. The first experiment was given one surface-irrigation 29 days after the establishment one at the tillering stage (water stress conditions, S). The second experiment was irrigated four times after sowing irrigation (normal conditions, N). In each experiment, the genotypes were grown in a randomized complete block design (RCBD) with three replicates. Each replicate consisted of 36 rows, $3 \mathrm{~m}$ long and $30 \mathrm{~cm}$ apart with $20 \mathrm{~cm}$ between plants. Sixteen grains were sown in each row and manually drilled in the rows. Each experiment was surrounded by a wide border $(12 \mathrm{~m})$ to minimize the underground water permeability. All other cultural practices, except irrigation, were applied as recommended for wheat cultivation. The two outside rows of the two external rows in each plot were discarded to eliminate the border effect.

Ten guarded plants for each parent and cross were tagged at random from each replication and data were recorded on heading date, maturity date, plant height, flag leaf area, spike length, number of spikelets per spike, number of spikes per plant number of the kernels for the highest spike, 1000 kernel weight and grain yield per 
plant. Monthly average temperature and amount of rainfall and mechanical and chemical analysis of experimental soil are shown in Tables (2) and (3).

Table (2): Meteorological data at Etay EL-Baroud location during 2010/11 growing season

\begin{tabular}{|c|c|c|c|c|c|c|c|c|c|}
\hline \multirow{3}{*}{ Month } & \multicolumn{4}{|c|}{ Air temperature $\left(\mathrm{C}^{0}\right)$} & \multirow{3}{*}{$\mathrm{RH} \%$} & \multirow{3}{*}{$\begin{array}{l}\text { Rain } \\
\text { fall } \\
(\mathrm{mm})\end{array}$} & \multicolumn{3}{|c|}{ Mean soil temperature $\left(\mathrm{C}^{\circ}\right)$} \\
\hline & \multicolumn{2}{|c|}{ Mean } & \multicolumn{2}{|c|}{ Extreme } & & & $5 \mathrm{~cm}$ & $10 \mathrm{~cm}$ & $20 \mathrm{~cm}$ \\
\hline & Max. & Min. & Max. & Min. & & & depth & depth & depth \\
\hline Oct. & 31 & 20.2 & 33 & 17 & 52.32 & 0 & 23.61 & 23.35 & 24.27 \\
\hline Nov. & 27.8 & 16.9 & 30 & 14 & 53.77 & 0 & 21.55 & 21.52 & 21.58 \\
\hline Dec. & 21.9 & 11.1 & 25 & 9 & 54.1 & 12 & 15.08 & 16.13 & 19.1 \\
\hline Jan. & 19.55 & 9.74 & 21 & 8 & 53.84 & 10 & 13.92 & 14.05 & 19.24 \\
\hline Feb. & 19.07 & 9.5 & 21 & 8 & 53.1 & 8 & 14.09 & 14.02 & 18.88 \\
\hline Mar. & 20.64 & 10.4 & 23 & 9 & 52.19 & 6 & 15.13 & 15.34 & 20.35 \\
\hline Apr. & 23.07 & 12.2 & 30 & 10 & 51.03 & 3 & 17.78 & 17.87 & 22.22 \\
\hline May & 25.42 & 13.5 & 29 & 12 & 49.6 & 0 & 19.13 & 19.34 & 22.4 \\
\hline
\end{tabular}

Table (3): Mechanical and chemical analysis of experimental soil in 2010/11 season at Etay EL-Baroud Research Station

\begin{tabular}{ll}
\hline \multicolumn{2}{c}{ Mechanical analysis } \\
\hline Clay \% & 52.6 \\
Silt \% & 26 \\
Sand \% & 21.4 \\
Textural class & Clay \\
\hline Chemical analysis & \\
\hline Available N mg/kg & 58.6 \\
Available P mg/kg & 7.8 \\
Available K mg/kg & 214 \\
\hline
\end{tabular}

The recorded data were subjected to the analysis of variance technique following Steel et al. (1997) to determine the significant differences among crosses and parents. General and specific combining ability estimates were estimated according to Griffing (1956) diallel cross analysis designated as method 2 model 1 for each experiment where genotypic differences were found significant. 


\section{REASULTES AND DISCUSSION}

\section{Analysis of variance and mean performance}

Analysis of variance for all studied traits for each of normal and stress environments are presented in Table (4).

Mean squares for genotypes, parents, crosses and parents vs. crosses were found to be significant for the ten measurements in both irrigation treatments indicating wide diversity between the parental genotypes used in the present study for these traits. Significant variations among genotypes for grain yield and related traits in different varieties of wheat were also reported by Ahmadi et al. (2003); Joshi et al. (2004); Hakim et al. (2007); Mohammadi et al. (2007); Seboka et al. (2009) and Saeed et al. (2010).

The mean performances of six wheat parental genotypes and their $\mathrm{F} 1$ at normal and stress irrigation conditions are presented in Table (5). The water stress treatment decreased the mean of days to heading and days to physiological maturity for parents and their hybrids. Line $1\left(\mathrm{P}_{6}\right)$ followed by Sakha $93\left(\mathrm{P}_{2}\right)$ were the earliest under the two irrigation conditions, while the parental genotype Sham $6\left(P_{5}\right)$ was the latest one. The two crosses $\left(P_{2} \times P_{6}\right)$ and $\left(P_{1} \times P_{6}\right)$ were the earliest in days to heading under both irrigation conditions. Meanwhile, the latest ones were the cross $\left(P_{1} \times P_{3}\right)$ under normal conditions, $\left(P_{3} \times P_{4}\right)$ and $\left(P_{3} \times P_{5}\right)$ under stress conditions. These results indicated that the parental genotype Line $1\left(P_{6}\right)$ possessed gene $(s)$ controlling earliness of days to heading, while parental genotypes Sham $6\left(P_{5}\right)$ and Gemmeiza $9\left(\mathrm{P}_{3}\right)$ have gene $(\mathrm{s})$ for lateness.

Regarding days to physiological maturity, among parents, the earliest in maturity were Line $1\left(\mathrm{P}_{6}\right)$ and Misr $1\left(\mathrm{P}_{4}\right)$ under both irrigation conditions, while Sham $6\left(P_{5}\right)$ was the latest one for this trait under the two irrigation conditions. Concerning crosses, three crosses $\left(P_{4} \times P_{6}\right),\left(P_{1} \times P_{6}\right)$ and $\left(P_{1} \times P_{4}\right)$ showed the lowest values (desirable) under both irrigation conditions.

For plant height, Sham $6\left(P_{5}\right)$ was the tallest stature followed by Sahel $1\left(P_{1}\right)$ and Gemmeiza $9\left(\mathrm{P}_{3}\right)$. However, Sakha $93\left(\mathrm{P}_{2}\right)$ was the shortest under the two irrigation conditions. Regarding crosses, two crosses $\left(P_{1} \times P_{2}\right)$ and $\left(P_{2} \times P_{4}\right)$ had the shortest plants under both irrigation conditions. However, crosses $\left(P_{3} \times P_{5}\right),\left(P_{5} \times P_{6}\right)$ and $\left(P_{3} \times P_{6}\right)$ had the highest mean values under normal irrigation meanwhile, crosses $\left(P_{1} \times P_{3}\right)$, $\left(P_{1} \times P_{5}\right)$, $\left(P_{1} \times P_{6}\right)$ and $\left(P_{3} \times P_{6}\right)$ showed the same performance under stress conditions. The reduction in plant height of stressed plants may be due to the reduction in internodes length and/or due to the reduction in moisture absorption, nutrient uptake and photosynthesis under drought stress condition.

The parents and crosses stressed for water had mean value of flag leaf area lower than plants under normal condition. For the parents, Line $1\left(\mathrm{P}_{6}\right)$ and Sham 6 $\left(P_{5}\right)$ were the highest for flag leaf area under both irrigation conditions. Concerning crosses, the three crosses $\left(P_{5} \times P_{6}\right),\left(P_{4} \times P_{6}\right)$ and $\left(P_{3} \times P_{5}\right)$ had the highest value under normal conditions, meanwhile crosses $\left(P_{4} \times P_{6}\right)$ and $\left(P_{5} \times P_{6}\right)$ were the highest under water stress conditions. Cross $\left(\mathrm{P}_{3} \times \mathrm{P}_{4}\right)$ had the lowest value of flag leaf area under both irrigation conditions. 
Table (4): Mean squares for the studied traits under each of normal $(\mathrm{N})$ and water stress (S) conditions

\begin{tabular}{|c|c|c|c|c|c|c|c|c|c|c|c|}
\hline \multirow[b]{2}{*}{ S.O.V. } & \multirow[b]{2}{*}{ d.f } & \multicolumn{2}{|c|}{ Days to heading } & \multicolumn{2}{|c|}{$\begin{array}{c}\text { Days to } \\
\text { physiological } \\
\text { maturity }\end{array}$} & \multicolumn{2}{|c|}{ Plant height } & \multicolumn{2}{|c|}{ Flag leaf area } & \multicolumn{2}{|c|}{ Spike length } \\
\hline & & $\mathrm{N}$ & $\mathrm{S}$ & $\mathrm{N}$ & $\mathrm{S}$ & $\mathrm{N}$ & $\mathrm{S}$ & $\mathrm{N}$ & $\mathrm{S}$ & $\mathrm{N}$ & $\mathrm{S}$ \\
\hline Rep. & 2 & 1.63 & 1.02 & 0.49 & 0.91 & 2.44 & 1.62 & 0.46 & 2.11 & 0.85 & 0.74 \\
\hline Genotypes & 20 & $58.74^{\star \star}$ & $43.76^{\star *}$ & $24.85^{\star *}$ & $35.71^{\star *}$ & $151.45^{\star *}$ & $107.26^{\star *}$ & $403.12^{* *}$ & $409.31^{\star *}$ & $7.70^{\star *}$ & $5.89^{* *}$ \\
\hline Parents & 5 & $148.46^{\star *}$ & $113.07^{* *}$ & $58.23^{\star *}$ & $49.96^{\star *}$ & $271.73^{\star *}$ & $146.97^{\star *}$ & $760.44^{\star *}$ & $836.91^{* *}$ & $13.57^{\star *}$ & $13.73^{* *}$ \\
\hline Error & 40 & 0.68 & 0.73 & 0.33 & 1.03 & 3.08 & 2.23 & 1.21 & 3.17 & 1.22 & 1.12 \\
\hline
\end{tabular}

** indicate significance at 0.01 levels of probability.

Table (4): Cont....

\begin{tabular}{|c|c|c|c|c|c|c|c|c|c|c|c|}
\hline \multirow[b]{2}{*}{ S.O.V. } & \multirow[b]{2}{*}{ d.f } & \multicolumn{2}{|c|}{$\begin{array}{c}\text { Number of } \\
\text { spikelets/spike }\end{array}$} & \multicolumn{2}{|c|}{$\begin{array}{c}\text { Number of } \\
\text { kernels/spike }\end{array}$} & \multicolumn{2}{|c|}{$\begin{array}{c}\text { Number of } \\
\text { spikes/ plant }\end{array}$} & \multicolumn{2}{|c|}{$\begin{array}{c}\text { 1000-kernel } \\
\text { weight }\end{array}$} & \multicolumn{2}{|c|}{ Grain yield/plant } \\
\hline & & $\mathrm{N}$ & S & $\mathrm{N}$ & S & $\mathrm{N}$ & $\mathrm{S}$ & $\mathrm{N}$ & S & $\mathrm{N}$ & $\mathrm{S}$ \\
\hline Rep. & 2 & 0.15 & 0.26 & 1.84 & 4.06 & 0.83 & 0.89 & 0.9 & 0.68 & 4.09 & 3.37 \\
\hline Genotypes & 20 & $3.17^{\star *}$ & $2.84^{\star \star}$ & $516.37^{\star *}$ & $502.77^{\star *}$ & $49.13^{\star \star}$ & $30.47^{* *}$ & $59.34^{\star *}$ & $90.36^{\star \star}$ & $226.65^{\star \star}$ & $142.01^{* *}$ \\
\hline Parents & 5 & $6.28^{* *}$ & $4.24^{\star \star}$ & $731.68^{\star *}$ & $631.59^{* *}$ & $69.18^{* *}$ & $35.08^{* *}$ & $97.01^{* *}$ & $128.94^{* *}$ & $254.12^{\star *}$ & 14.52 \\
\hline Crosses & 14 & $2.19^{\star *}$ & $2.46^{\star \star}$ & $467.92^{\star \star}$ & $485.97^{\star *}$ & $37.82^{\star \star}$ & $28.23^{\star *}$ & $28.22^{\star \star}$ & $63.08^{\star *}$ & $62.23^{\star \star}$ & $127.01^{* *}$ \\
\hline P vs. C & 1 & $1.33^{\star \star}$ & $1.18^{\star}$ & $118.27^{\star \star}$ & $93.92^{\star \star}$ & $107.13^{\star *}$ & $38.90^{* *}$ & $306.67^{* \star}$ & $279.36^{\star *}$ & $2391.17^{\star \star}$ & $989.48^{* \star}$ \\
\hline Error & 40 & 0.39 & 0.48 & 3.74 & 5.45 & 1.29 & 1.27 & 1.03 & 0.45 & 10.02 & 10.5 \\
\hline
\end{tabular}


Regarding spike length, Line $1\left(\mathrm{P}_{6}\right)$ had the tallest spike under the two irrigation conditions. Cross $\left(\mathrm{P}_{5} \times \mathrm{P}_{6}\right)$ had the tallest spike under normal conditions followed by four crosses i.e. $\left.\left(P_{3} \times P_{6}\right), P_{4} \times P_{6}\right),\left(P_{2} \times P_{6}\right)$ and $\left(P_{2} \times P_{3}\right)$. Meanwhile crosses $\left(P_{4} \times P_{6}\right),\left(P_{2} \times P_{6}\right),\left(P_{3} \times P_{6}\right)$ and $\left(P_{1} \times P_{6}\right)$, respectively had the tallest spike under stress conditions, cross $\left(\mathrm{P}_{3} \times \mathrm{P}_{4}\right)$ showed the lowest value for this trait under both conditions.

For number of spikelets per spike, Gemmeiza $9\left(P_{3}\right)$ followed by Sahel $1\left(P_{1}\right)$ and Line $1\left(P_{6}\right)$ were the highest among parents under both irrigation conditions. Crosses $\left(P_{1} \times P_{3}\right)$ and $\left(P_{3} \times P_{6}\right)$ had the highest number of spikelets per spike under normal conditions. On the other hand, crosses $\left(P_{1} \times P_{3}\right),\left(P_{1} \times P_{6}\right),\left(P_{2} \times P_{3}\right),\left(P_{3} \times P_{5}\right)$ and $\left(\mathrm{P}_{3} \times \mathrm{P}_{6}\right)$ exhibited the highest one under stress conditions.

Concerning number of grains per spike, Line $1\left(\mathrm{P}_{6}\right)$ ranked the first under both irrigation conditions; the two crosses $\left(P_{1} \times P_{6} \& P_{4} \times P_{6}\right)$ ranked the first and the second respectively, under both irrigation conditions followed by $\left(P_{5} \times P_{6}\right)$ and $\left(P_{2} \times\right.$ $P_{6}$ ) under normal conditions. The high number of grains per spike in these crosses cold be attributed to superiority of the parent Line $1\left(\mathrm{P}_{6}\right)$ in this trait. It is clear from the data that water stress treatment decreased the mean of number of grains per spike for parents and their hybrids. This reduction may be due to the effect of water deficit on pollination and fertilization processes, which lead to decreasing number of grains per spike.

Concerning number of spikes per plant, the two parents Shame $6\left(P_{5}\right)$ and Misr $1\left(\mathrm{P}_{4}\right)$ owned the highest number under both irrigation conditions. Meanwhile, the parent Line $1\left(\mathrm{P}_{6}\right)$ owned the fewest number of spikes per plant. Under normal conditions cross $\left(\mathrm{P}_{2} \times \mathrm{P}_{4}\right)$ owned the highest number followed by cross $\left(\mathrm{P}_{1} \times \mathrm{P}_{2}\right)$.

For 1000-kernel weight, the parental genotype Line $1\left(P_{6}\right)$ was the superior parent under both irrigation conditions. All the five crosses that contain the parent Line $1\left(\mathrm{P}_{6}\right)$ in addition to the two crosses $\left(\mathrm{P}_{3} \times \mathrm{P}_{4}\right.$ and $\left.\mathrm{P}_{2} \times \mathrm{P}_{4}\right)$ exhibited the highest values for this trait under normal conditions, cross $\left(P_{3} \times P_{6}\right)$ ranked the first under both conditions. It's clear that the parental genotype Line $1\left(P_{6}\right)$ possess gene (s) controlling high kernel weight. The high 1000-kernel weight in this parent could be attributed to its high flag leaf area.

Concerning grain yield per plant, the parent Misr $1\left(\mathrm{P}_{4}\right)$ showed the highest value of grain yield per plant under normal conditions. Meanwhile, the parent Sham 6 $\left(P_{5}\right)$ had the highest value of grain yield per plant under stress conditions. Under normal conditions crosses $\left(P_{1} \times P_{3}\right)$ and $\left(P_{3} \times P_{6}\right)$ ranked first and second respectively, followed by the two crosses $\left(P_{2} \times P_{3}\right)$ and $\left(P_{2} \times P_{6}\right)$ under both irrigation conditions. 
Table (5): The genotypes mean performance for the studied characters under normal (N) and water stress (S) conditions

\begin{tabular}{|c|c|c|c|c|c|c|c|c|c|c|}
\hline \multirow[t]{2}{*}{ Genotypes } & \multicolumn{2}{|c|}{$\begin{array}{l}\text { Days to } \\
\text { heading } \\
\text { (days) }\end{array}$} & \multicolumn{2}{|c|}{$\begin{array}{c}\text { Days to } \\
\text { physiological } \\
\text { maturity (days) }\end{array}$} & \multicolumn{2}{|c|}{$\begin{array}{l}\text { Plant height } \\
\text { (cm) }\end{array}$} & \multicolumn{2}{|c|}{$\begin{array}{c}\text { Flag leaf area } \\
\left(\mathrm{cm}^{2}\right)\end{array}$} & \multicolumn{2}{|c|}{$\begin{array}{l}\text { Spike length } \\
\text { (cm) }\end{array}$} \\
\hline & $\mathrm{N}$ & $\mathrm{S}$ & $\mathrm{N}$ & $\mathrm{S}$ & $\mathrm{N}$ & $\mathrm{S}$ & $\mathrm{N}$ & $\mathrm{S}$ & $\mathrm{N}$ & $\mathrm{S}$ \\
\hline Sahel $1\left(P_{1}\right)$ & 102.00 & 95.33 & 152.33 & 140.00 & 107.10 & 97.58 & 59.97 & 48.20 & 13.43 & 12.42 \\
\hline Sakha $93\left(P_{2}\right)$ & 93.00 & 90.67 & 151.33 & 143.67 & 84.42 & 79.58 & 68.15 & 62.53 & 13.67 & 12.94 \\
\hline Gemmeiza $9\left(P_{3}\right)$ & 104.33 & 99.00 & 151.33 & 143.33 & 105.79 & 94.30 & 51.11 & 35.54 & 15.02 & 13.53 \\
\hline Misr $1\left(P_{4}\right)$ & 96.33 & 93.00 & 147.00 & 137.00 & 97.17 & 88.82 & 59.94 & 44.85 & 13.13 & 12.63 \\
\hline Sham $6\left(P_{5}\right)$ & 106.00 & 103.67 & 153.33 & 144.33 & 110.70 & 98.67 & 88.05 & 62.13 & 15.15 & 14.50 \\
\hline Line $1\left(P_{6}\right)$ & 88.00 & 86.33 & 141.67 & 134.33 & 97.73 & 91.89 & 89.53 & 82.67 & 18.87 & 18.11 \\
\hline $1 \times 2$ & 93.67 & 90.00 & 151.67 & 142.67 & 94.30 & 89.43 & 70.32 & 68.67 & 14.69 & 13.86 \\
\hline $1 \times 3$ & 100.67 & 93.33 & 149.67 & 141.33 & 106.88 & 105.08 & 56.05 & 54.55 & 15.02 & 13.92 \\
\hline $1 \times 4$ & 95.67 & 89.67 & 147.33 & 135.67 & 103.11 & 96.00 & 76.60 & 67.70 & 14.50 & 13.80 \\
\hline $1 \times 5$ & 95.00 & 89.00 & 148.67 & 137.67 & 104.90 & 103.00 & 72.64 & 72.19 & 14.65 & 14.82 \\
\hline $1 \times 6$ & 92.67 & 88.67 & 146.00 & 135.00 & 106.20 & 102.36 & 71.99 & 51.95 & 16.40 & 15.56 \\
\hline $2 \times 3$ & 93.00 & 91.67 & 149.67 & 144.33 & 100.00 & 93.00 & 69.80 & 67.50 & 14.50 & 14.03 \\
\hline $2 \times 4$ & 93.67 & 91.67 & 150.67 & 137.33 & 97.00 & 88.10 & 71.91 & 60.30 & 13.59 & 13.11 \\
\hline $2 \times 5$ & 95.67 & 91.67 & 150.33 & 144.00 & 100.00 & 94.35 & 76.12 & 67.78 & 14.80 & 14.15 \\
\hline $2 \times 6$ & 90.33 & 88.33 & 146.67 & 142.00 & 100.35 & 94.23 & 79.00 & 71.79 & 16.89 & 16.10 \\
\hline $3 \times 4$ & 97.67 & 94.67 & 148.33 & 139.00 & 107.87 & 93.00 & 56.66 & 47.00 & 13.97 & 12.90 \\
\hline $3 \times 5$ & 97.67 & 94.67 & 152.33 & 140.33 & 113.23 & 98.40 & 81.05 & 63.15 & 15.50 & 13.85 \\
\hline $3 \times 6$ & 95.67 & 93.33 & 147.33 & 137.67 & 111.30 & 102.30 & 66.23 & 64.60 & 17.41 & 15.85 \\
\hline $4 \times 5$ & 97.00 & 92.67 & 149.33 & 137.33 & 109.58 & 99.25 & 66.74 & 64.31 & 14.57 & 14.10 \\
\hline $4 \times 6$ & 92.33 & 90.67 & 145.00 & 133.67 & 108.30 & 99.39 & 83.73 & 77.00 & 17.00 & 16.27 \\
\hline $5 \times 6$ & 93.33 & 91.67 & 146.33 & 140.00 & 112.82 & 96.90 & 94.72 & 74.50 & 18.29 & 14.85 \\
\hline Mean of parents & 98.28 & 94.67 & 149.50 & 140.44 & 100.49 & 91.81 & 69.46 & 55.99 & 14.88 & 14.02 \\
\hline Mean of crosses & 94.93 & 91.44 & 148.62 & 139.20 & 105.06 & 96.99 & 72.90 & 64.87 & 15.45 & 14.48 \\
\hline Mean of genotypes & 95.89 & 92.37 & 148.87 & 139.56 & 103.75 & 95.51 & 71.92 & 62.33 & 15.29 & 14.35 \\
\hline L.S.D. at $5 \%$ & 1.36 & 1.41 & 0.94 & 1.67 & 2.90 & 2.46 & 1.81 & 2.94 & 0.56 & 0.57 \\
\hline L.S.D. at $1 \%$ & 1.82 & 1.89 & 1.26 & 2.24 & 3.88 & 3.29 & 2.43 & 3.93 & 0.75 & 0.77 \\
\hline
\end{tabular}


J. Adv. Agric. Res. (Fac. Agric. Saba Basha)

Table (5): Cont....

\begin{tabular}{|c|c|c|c|c|c|c|c|c|c|c|}
\hline \multirow[t]{2}{*}{ Genotypes } & \multicolumn{2}{|c|}{$\begin{array}{c}\text { Number } \\
\text { of } \\
\text { spikelets/spike }\end{array}$} & \multicolumn{2}{|c|}{$\begin{array}{c}\text { Number } \\
\text { of } \\
\text { kernels/spike }\end{array}$} & \multicolumn{2}{|c|}{$\begin{array}{c}\text { Number } \\
\text { of } \\
\text { spikes/plant } \\
\end{array}$} & \multicolumn{2}{|c|}{$\begin{array}{c}\text { 1000-kernel } \\
\text { weight } \\
(\mathrm{g})\end{array}$} & \multicolumn{2}{|c|}{$\begin{array}{c}\text { Grain } \\
\text { yield/plant (g) }\end{array}$} \\
\hline & $\mathrm{N}$ & $\mathrm{S}$ & $\mathrm{N}$ & $\mathrm{S}$ & $\mathrm{N}$ & $\mathrm{S}$ & $\mathrm{N}$ & $\mathrm{S}$ & $\mathrm{N}$ & S \\
\hline Sahel $1\left(P_{1}\right)$ & 26.31 & 24.21 & 95.67 & 89.67 & 12.92 & 10.42 & 40.48 & 37.22 & 38.89 & 32.09 \\
\hline Sakha $93\left(P_{2}\right)$ & 24.48 & 23.70 & 80.44 & 75.80 & 13.78 & 11.77 & 43.93 & 37.37 & 37.77 & 34.50 \\
\hline Gemmeiza $9\left(P_{3}\right)$ & 28.00 & 26.36 & 95.29 & 81.18 & 13.31 & 9.33 & 45.63 & 43.35 & 48.02 & 30.85 \\
\hline Misr $1\left(P_{4}\right)$ & 24.20 & 22.97 & 85.83 & 79.22 & 17.14 & 13.10 & 43.80 & 38.97 & 58.77 & 33.05 \\
\hline Sham $6\left(P_{5}\right)$ & 24.80 & 24.31 & 84.29 & 78.00 & 17.75 & 13.33 & 46.67 & 39.37 & 48.00 & 36.22 \\
\hline Line $1\left(P_{6}\right)$ & 26.22 & 25.21 & 123.50 & 114.33 & 4.33 & 4.11 & 57.04 & 54.37 & 33.58 & 30.50 \\
\hline $1 \times 2$ & 26.24 & 24.90 & 89.42 & 83.91 & 20.30 & 14.95 & 47.75 & 39.80 & 56.50 & 45.40 \\
\hline $1 \times 3$ & 27.87 & 25.94 & 92.38 & 81.80 & 17.00 & 13.75 & 49.15 & 45.25 & 65.00 & 41.00 \\
\hline $1 \times 4$ & 24.96 & 24.35 & 85.98 & 78.00 & 17.68 & 15.35 & 48.85 & 45.10 & 57.35 & 49.14 \\
\hline $1 \times 5$ & 24.93 & 24.22 & 82.07 & 86.21 & 17.75 & 15.35 & 47.35 & 42.50 & 57.53 & 46.09 \\
\hline $1 \times 6$ & 26.70 & 25.92 & 117.50 & 113.67 & 10.33 & 8.01 & 51.40 & 49.30 & 59.70 & 46.67 \\
\hline $2 \times 3$ & 26.30 & 25.81 & 90.37 & 80.00 & 17.67 & 15.33 & 49.65 & 46.25 & 61.73 & 50.10 \\
\hline $2 \times 4$ & 24.93 & 22.90 & 77.03 & 67.67 & 23.50 & 13.68 & 51.05 & 41.35 & 57.26 & 42.73 \\
\hline $2 \times 5$ & 25.03 & 24.22 & 78.18 & 73.16 & 16.00 & 12.75 & 49.45 & 41.70 & 45.25 & 39.00 \\
\hline $2 \times 6$ & 26.38 & 25.04 & 99.56 & 90.00 & 13.52 & 12.10 & 56.50 & 49.25 & 60.00 & 49.47 \\
\hline $3 \times 4$ & 25.87 & 24.50 & 80.34 & 69.69 & 15.55 & 13.76 & 53.80 & 51.59 & 52.00 & 37.90 \\
\hline $3 \times 5$ & 26.11 & 25.56 & 89.62 & 77.00 & 17.62 & 10.48 & 50.85 & 42.62 & 59.53 & 33.50 \\
\hline $3 \times 6$ & 27.14 & 25.50 & 89.50 & 85.72 & 13.50 & 7.45 & 58.35 & 56.40 & 62.07 & 31.00 \\
\hline $4 \times 5$ & 25.54 & 23.40 & 77.57 & 73.56 & 17.73 & 13.21 & 49.50 & 45.40 & 57.95 & 38.09 \\
\hline $4 \times 6$ & 26.13 & 24.89 & 110.97 & 107.00 & 10.30 & 7.65 & 52.05 & 50.40 & 57.75 & 44.00 \\
\hline $5 \times 6$ & 25.70 & 24.30 & 106.56 & 87.57 & 12.94 & 7.45 & 51.45 & 49.60 & 57.50 & 30.50 \\
\hline Mean of parents & 25.67 & 24.46 & 94.17 & 86.37 & 13.21 & 10.35 & 46.26 & 41.77 & 44.17 & 32.87 \\
\hline Mean of crosses & 25.99 & 24.76 & 91.14 & 83.66 & 16.09 & 12.08 & 51.14 & 46.43 & 57.81 & 41.64 \\
\hline Mean of genotypes & 25.90 & 24.68 & 92.00 & 84.44 & 15.27 & 11.59 & 49.75 & 45.10 & 53.91 & 39.13 \\
\hline L.S.D. at $5 \%$ & 1.87 & 1.86 & 3.19 & 3.85 & 1.87 & 1.86 & 1.67 & 1.11 & 5.22 & 5.35 \\
\hline L.S.D. at $1 \%$ & 2.51 & 2.48 & 4.27 & 5.15 & 2.51 & 2.48 & 2.24 & 1.49 & 6.99 & 7.15 \\
\hline
\end{tabular}




\section{Combining ability}

Analysis of variance for combining ability in normal and stress conditions is presented in Table (6).Mean squares associated with general combining ability (GCA) and specific combing ability (SCA) were found to be significant for all studied measurements in both irrigation conditions. It is evident that additive type of gene action was more important part of the total genetic variability for these traits in both normal and stress irrigation conditions. High GCA/SCA ratios which largely exceeded the unity were detected for all traits, except for grain yield/plant under the study in both irrigation treatments, such results indicated the predominance of additive and additive $x$ additive types of gene action in the inheritance of such traits. These results are in agreement with those found by Abd Elnour (2005). Significant differences due to general combining ability and specific combining ability were observed for the studied traits (Pang et al., 2010).

\section{General combining ability effects:}

General combining ability effects $\left(\hat{g}_{i}\right)$ for individual parental line and/or cultivar for all studied measurements at normal and stress irrigation conditions are presented in Table (7). Such results are being used to compare the average performance of each parent with other genotype and facilitate selection of parents for further improvement to drought resistance. High positive values would be of interest for all measurements in question except days to heading and days to physiological maturity where high negative effects would be useful from the breeder point of view.

The two parental genotypes Misr $1\left(\mathrm{P}_{4}\right)$ and Line $1\left(\mathrm{P}_{6}\right)$ expressed significant negative $\left(\hat{g}_{i}\right)$ effects for days to heading and days to physiological maturity under both normal and stress conditions, indicating that both parental genotypes could be considered as a good combiners for developing early genotypes.

For plant height, under the two irrigation conditions significant negative $\left(\hat{g}_{i}\right)$ effects were detected for Sakha $93\left(\mathrm{P}_{2}\right)$ and Misr $1\left(\mathrm{P}_{4}\right)$ respectively, revealing the possibility of utilizing these parents to release short stature varieties. On the other hand, considerable significant or highly significant positive values were detected for the other parents under the two irrigation conditions, except Sahel $1\left(P_{1}\right)$ at normal irrigation conditions, showing that these genotypes are suitable in breeding programs towards releasing varieties for higher plant height under the previous conditions. Yet releasing cultivars with short plants may be of special interest for such purpose.

Regarding flag leaf area, highly significant positive GCA effects were detected by Sham $6\left(P_{5}\right)$ and Line $1\left(P_{6}\right)$ at both conditions and Sakha $93\left(P_{2}\right)$ under normal condition for showing that these parents appeared to be good combiners for this trait at the previous conditions. The other four parents gave significant negative or in significant $\left(\hat{g}_{i}\right)$ effects for this trait. 
Table (6): Mean squares of general and specific combining ability from diallel cross analysis for all studied traits under normal $(\mathrm{N})$ and water stress $(\mathrm{S})$ conditions

\begin{tabular}{|c|c|c|c|c|c|c|c|c|c|c|c|}
\hline \multirow[t]{2}{*}{ S.O.V. } & \multirow[t]{2}{*}{ d.f } & \multicolumn{2}{|c|}{ Days to heading } & \multicolumn{2}{|c|}{$\begin{array}{l}\text { Days to } \\
\text { physiological } \\
\text { maturity }\end{array}$} & \multicolumn{2}{|c|}{ Plant height } & \multicolumn{2}{|c|}{ Flag leaf area } & \multicolumn{2}{|c|}{ Spike length } \\
\hline & & $\mathrm{N}$ & $\mathrm{S}$ & $\mathrm{N}$ & S & $\mathrm{N}$ & $\mathrm{S}$ & $\mathrm{N}$ & $S$ & $\mathrm{~N}$ & $\mathrm{~S}$ \\
\hline Geno. & 20 & $58.74^{* *}$ & $43.76^{\star \star}$ & $24.85^{\star *}$ & $35.71^{* *}$ & $151.45^{\star *}$ & $107.26^{* *}$ & $403.12^{* *}$ & $409.31^{\star *}$ & $7.70^{\star *}$ & $5.89^{* *}$ \\
\hline GCA & 5 & $57.30^{* *}$ & $35.09^{* *}$ & $28.26^{* *}$ & $38.05^{* *}$ & $157.94^{\star *}$ & $98.49^{* *}$ & $408.46^{\star *}$ & $311.65^{\star *}$ & $9.56^{* *}$ & $6.63^{* *}$ \\
\hline SCA & 15 & $7.01^{\star *}$ & $7.75^{\star \star}$ & $1.62^{\star \star}$ & $3.19^{\star *}$ & $14.67^{\star \star}$ & $14.84^{\star *}$ & $43.01^{\star *}$ & $78.03^{\star \star}$ & $0.24^{\star *}$ & $0.41^{\star *}$ \\
\hline GCA/SCS & & 8.17 & 4.53 & 17.4 & 11.93 & 10.77 & 6.64 & 9.5 & 3.99 & 40.51 & 16.19 \\
\hline Error term & 40 & 0.23 & 0.24 & 0.11 & 0.34 & 1.03 & 0.74 & 0.4 & 1.06 & 0.04 & 0.04 \\
\hline
\end{tabular}

Table (6): Cont....

\begin{tabular}{|c|c|c|c|c|c|c|c|c|c|c|c|}
\hline \multirow[t]{2}{*}{ S.O.V. } & \multirow[t]{2}{*}{ d.f } & \multicolumn{2}{|c|}{$\begin{array}{c}\text { Number of } \\
\text { spikelets/spike }\end{array}$} & \multicolumn{2}{|c|}{$\begin{array}{c}\text { Number of } \\
\text { kernels/spike }\end{array}$} & \multicolumn{2}{|c|}{$\begin{array}{l}\text { Number of } \\
\text { spikes/plant }\end{array}$} & \multicolumn{2}{|c|}{$\begin{array}{c}\text { 1000-kernel } \\
\text { weight }\end{array}$} & \multicolumn{2}{|c|}{ Grain yield/plant } \\
\hline & & $\mathrm{N}$ & $S$ & $\mathrm{~N}$ & $S$ & $\mathrm{~N}$ & $S$ & $\mathrm{~N}$ & $S$ & $\mathrm{~N}$ & $S$ \\
\hline Geno. & 20 & $3.17^{\star \star}$ & $2.84^{* \star}$ & $516.37^{* *}$ & $502.77^{\star *}$ & $49.13^{\star *}$ & $30.47^{* *}$ & $59.34^{\star *}$ & $90.36^{* *}$ & $226.65^{\star *}$ & $142.01^{* *}$ \\
\hline GCA & 5 & $3.48^{\star *}$ & $3.08^{* *}$ & $555.08^{* *}$ & $543.49^{* *}$ & $45.47^{\star *}$ & $29.84^{* *}$ & $47.84^{\star *}$ & $90.09^{* *}$ & $37.75^{\star \star}$ & $37.95^{\star *}$ \\
\hline SCA & 15 & $0.25^{\star *}$ & $0.24^{\star *}$ & $44.47^{* *}$ & $42.29^{\star \star}$ & $6.68^{* *}$ & $3.60^{* *}$ & $10.43^{* *}$ & $10.13^{* *}$ & $88.15^{\star *}$ & $50.46^{* *}$ \\
\hline GCA/SCA & & 13.93 & 12.96 & 12.48 & 12.85 & 6.81 & 8.3 & 4.59 & 8.89 & 0.43 & 0.75 \\
\hline Error term & 40 & 0.03 & 0.06 & 1.25 & 1.82 & 0.43 & 0.42 & 0.34 & 0.15 & 3.34 & 3.5 \\
\hline
\end{tabular}

** indicate significance at 0.01 levels of probability.

GCA refers to general combining ability.

SCA refers to specific combining ability. 
Concerning spike length, only two parents i.e. Sham $6\left(P_{5}\right)$ and Line $1\left(P_{6}\right)$ exhibited highly significant and significant positive $\left(\hat{g}_{i}\right)$ effects under normal irrigation conditions only. Meanwhile, the other parents gave highly significant negative or in significant $\left(\hat{g}_{i}\right)$ effects for this trait.

For number of spikelets/spike, under both normal and water stress conditions, the parental genotypes Gemmeiza $9\left(\mathrm{P}_{3}\right)$ and Line $1\left(\mathrm{P}_{6}\right)$ gave the desirable $\left(\hat{g}_{i}\right)$ effects. Meanwhile, the parent Sahel $1\left(P_{1}\right)$ showed the same result under normal irrigation condition only. The other parental genotypes gave undesirable $\left(\hat{g}_{i}\right)$ effect under both irrigation conditions for this trait.

Regarding number of grains/spike, the parental Line $1\left(\mathrm{P}_{6}\right)$ was the best combiner of number of grains/spike, it gave the highest significant positive $\left(\hat{g}_{i}\right)$ effects under the two irrigation conditions followed by the parental genotype Sahel 1 $\left(P_{1}\right)$. Therefore, they could be considered as the best combiners for this trait.

Regarding number of spikes/plant, the parental Sakha $93\left(\mathrm{P}_{2}\right)$, Misr $1\left(\mathrm{P}_{4}\right)$ and Sham $6\left(\mathrm{P}_{5}\right)$ showed the desirable $\left(\hat{g}_{i}\right)$ effects under normal conditions, in addition to the previous parents the parent Sahel $1\left(\mathrm{P}_{1}\right)$ showed the desirable $\left(\hat{g}_{i}\right)$ effects under stress conditions. The parental Line $1\left(\mathrm{P}_{6}\right)$ was the inferior for this trait and appeared to be bad combiner for this trait at the previous conditions.

For 1000- kernel weight, two parental genotypes i.e. Gemmeiza $9\left(P_{3}\right)$ and Line $1\left(\mathrm{P}_{6}\right)$ expressed highly significant positive $\left(\hat{g}_{i}\right)$ effects under both irrigation conditions. However, Line $1\left(P_{6}\right)$ was the superior one indicating that these genotypes could be considered as good combiners for this trait. While, Sahel $1\left(P_{1}\right)$, Sakha 93 $\left(P_{2}\right)$, Misr $1\left(P_{4}\right)$ and Sham $6\left(P_{5}\right)$ showed highly significant negative effects in both irrigation conditions, suggesting that these genotypes could not be considered as good combiners for developing this trait.

The estimates of GCA effects for grain yield per plant are highly significant and positive in Gemmeiza $9\left(\mathrm{P}_{3}\right)$ and Misr $1\left(\mathrm{P}_{4}\right)$ under normal irrigation conditions. It could be noticed that Sahel $1\left(\mathrm{P}_{1}\right)$ and Sakha $93\left(\mathrm{P}_{2}\right)$ indicted desirable $\left(\hat{g}_{i}\right)$ under water stress conditions. Negative and significant estimates of GCA effects were obtained for Line $1\left(P_{6}\right)$ under the two irrigation conditions. These results are partially in harmony with Sultan et al., (2011) who pointed to the importance of additive gene effects in the inheritance of plant height and spikes number plant ${ }^{-1}$, while, additive, dominance and epistasis were the important in the inheritance of grains number spike $^{-1}, 100$-grain weight and grain yield plant ${ }^{-1}$ at most cases under both normal and water stress conditions. Moreover, additive genetic variance played the greatest and the important role in the inheritance of plant height, spikes number plant ${ }^{-1}$ and grain

Vol. $19(4), 2014$ 
Table (7): Estimation of general combining ability (GCA) effects for studied parents under normal (N) and water stress (S) conditions

\begin{tabular}{|c|c|c|c|c|c|c|c|c|c|c|}
\hline \multirow{2}{*}{$\begin{array}{l}\text { Traits } \\
\text { Parents }\end{array}$} & \multicolumn{2}{|c|}{ Days to heading } & \multicolumn{2}{|c|}{$\begin{array}{c}\text { Days to } \\
\text { physiological } \\
\text { maturity }\end{array}$} & \multicolumn{2}{|c|}{ Plant height } & \multicolumn{2}{|c|}{ Flag leaf area } & \multicolumn{2}{|c|}{ Spike length } \\
\hline & $\mathrm{N}$ & $S$ & $\mathrm{~N}$ & $\mathrm{~S}$ & $\mathrm{~N}$ & $S$ & $\mathrm{~N}$ & $\mathrm{~S}$ & $\mathrm{~N}$ & $S$ \\
\hline P1 (Sahel 1) & $1.31^{\star \star}$ & $-0.65^{\star \star}$ & $0.74^{\star \star}$ & $-0.57^{\star \star}$ & 0.42 & $2.81^{\star \star}$ & $-4.49^{\star \star}$ & $-3.11^{\star *}$ & $-0.61^{\star \star}$ & $-0.46^{\star \star}$ \\
\hline P2 (Sakha 93) & $-2.36^{\star *}$ & $-1.49^{* *}$ & $1.19^{\star *}$ & $2.60^{* *}$ & $-8.22^{\star *}$ & $-6.28^{* *}$ & 0.00 & $3.10^{* *}$ & $-0.65^{\star *}$ & $-0.41^{* *}$ \\
\hline P3 (Gemmeiza 9) & $2.76^{\star \star}$ & $2.39^{\star *}$ & $0.99^{* *}$ & $1.56^{\star \star}$ & $3.08^{* *}$ & $1.48^{* \star}$ & $-8.93^{\star *}$ & $-8.55^{\star \star}$ & -0.07 & $-0.35^{\star *}$ \\
\hline P4 (Misr 1) & $-0.28^{\star *}$ & -0.15 & $-0.93^{\star *}$ & $-2.49^{* *}$ & $-0.76^{*}$ & $-1.90^{* *}$ & $-3.49^{* *}$ & $-3.79^{\star *}$ & $-0.89^{* *}$ & $-0.62^{\star *}$ \\
\hline P5 (Sham 6) & $2.43^{\star \star}$ & $2.56^{\star \star}$ & $1.44^{\star \star}$ & $1.39^{\star *}$ & $4.46^{\star \star}$ & $2.59^{\star *}$ & $7.99^{\star *}$ & $3.73^{\star \star}$ & $0.14^{*}$ & 0.04 \\
\hline P6 (Line 1) & $-3.86^{\star \star}$ & $-2.65^{\star \star}$ & $-3.43^{\star \star}$ & $-2.49^{* *}$ & $1.02^{\star \star}$ & $1.30^{* *}$ & $8.91^{\star *}$ & $8.61^{* *}$ & $2.09^{\star *}$ & $1.80^{\star \star}$ \\
\hline $\operatorname{LSD}\left(g_{i}\right)_{0.05}$ & 0.31 & 0.32 & 0.21 & 0.38 & 0.66 & 0.56 & 0.41 & 0.67 & 0.13 & 0.13 \\
\hline $\operatorname{LSD}\left(g_{i}\right)_{0.01}$ & 0.42 & 0.43 & 0.29 & 0.51 & 0.88 & 0.75 & 0.55 & 0.9 & 0.17 & 0.17 \\
\hline LSD $\left(g_{\mathrm{i}}-g_{\mathrm{j}}\right)_{0.05}$ & 0.48 & 0.5 & 0.33 & 0.59 & 1.02 & 0.87 & 0.64 & 1.04 & 0.2 & 0.2 \\
\hline LSD $\left(g_{i}-g_{j}\right)_{0.01}$ & 0.64 & 0.67 & 0.45 & 0.79 & 1.37 & 1.16 & 0.86 & 1.39 & 0.26 & 0.27 \\
\hline
\end{tabular}

Table (7): Cont....

\begin{tabular}{|c|c|c|c|c|c|c|c|c|c|c|}
\hline \multirow{2}{*}{$\begin{array}{l}\text { Traits } \\
\text { Parents }\end{array}$} & \multicolumn{2}{|c|}{$\begin{array}{c}\text { Number of } \\
\text { spikelets/spike }\end{array}$} & \multicolumn{2}{|c|}{$\begin{array}{c}\text { Number of } \\
\text { kernels/spike }\end{array}$} & \multicolumn{2}{|c|}{$\begin{array}{c}\text { Number of } \\
\text { spikes/plant }\end{array}$} & \multicolumn{2}{|c|}{$\begin{array}{c}\text { 1000-kernel } \\
\text { weight }\end{array}$} & \multicolumn{2}{|c|}{ Grain yield/plant } \\
\hline & $\mathrm{N}$ & S & $\mathrm{N}$ & S & $\mathrm{N}$ & S & $\mathrm{N}$ & $\mathrm{S}$ & $\mathrm{N}$ & $\mathrm{S}$ \\
\hline P1 (Sahel 1) & $0.26^{\star \star}$ & 0.13 & $1.83^{\star *}$ & $3.98^{\star \star}$ & 0.25 & $0.89^{\star \star}$ & $-2.85^{\star *}$ & $-2.42^{\star *}$ & -0.44 & $2.32^{\star *}$ \\
\hline P2 (Sakha 93) & $-0.43^{\star *}$ & $-0.31^{* *}$ & $-6.07^{\star \star}$ & $-5.59^{\star \star}$ & $1.46^{\star *}$ & $1.41^{\star \star}$ & $-0.75^{\star *}$ & $-2.83^{* *}$ & $-2.64^{* *}$ & $2.72^{\star \star}$ \\
\hline P3 (Gemmeiza 9) & $1.00^{* *}$ & $0.91^{* *}$ & $-1.40^{\star *}$ & $-4.31^{* *}$ & 0.13 & -0.21 & $0.60^{* *}$ & $1.64^{\star *}$ & $2.37^{\star \star *}$ & $-2.34^{\star \star}$ \\
\hline P4 (Misr 1) & $-0.68^{\star *}$ & $-0.85^{\star \star}$ & $-5.06^{\star *}$ & $-4.59^{\star *}$ & $1.52^{\star \star}$ & $1.09^{\star *}$ & $-0.67^{\star *}$ & $-0.49^{* *}$ & $2.81^{\star *}$ & 0.5 \\
\hline P5 (Sham 6) & $-0.55^{\star *}$ & $-0.30^{* *}$ & $-5.18^{\star *}$ & $-4.69^{* *}$ & $1.33^{\star *}$ & $0.60^{* *}$ & -0.79 ** & $-1.90^{* *}$ & -0.45 & $-1.79^{* \star}$ \\
\hline P6 (Line 1) & $0.40^{\star *}$ & $0.42^{* *}$ & $15.88^{\star *}$ & $15.20^{* *}$ & $-4.70^{\star *}$ & $-3.78^{* *}$ & $4.45^{\star *}$ & $6.00^{* *}$ & $-1.65^{\star *}$ & $-1.41^{*}$ \\
\hline LSD $\left(g_{i}\right)_{0.05}$ & 0.11 & 0.16 & 0.73 & 0.88 & 0.43 & 0.42 & 0.38 & 0.25 & 1.19 & 1.22 \\
\hline LSD $\left(g_{i}\right)_{0.01}$ & 0.15 & 0.22 & 0.97 & 1.18 & 0.57 & 0.57 & 0.51 & 0.34 & 1.6 & 1.63 \\
\hline LSD $\left(g_{i}-g_{j}\right)_{0.05}$ & 0.18 & 0.25 & 1.13 & 1.36 & 0.66 & 0.66 & 0.59 & 0.39 & 1.85 & 1.89 \\
\hline LSD $\left(g_{i}-g_{j}\right)_{0.01}$ & 0.24 & 0.33 & 1.51 & 1.82 & 0.89 & 0.88 & 0.79 & 0.53 & 2.47 & 2.53 \\
\hline
\end{tabular}


yield plant $^{-1}$ at most cases under both water conditions. On the other hand, dominance genetic variance was the greatest and the important in the inheritance of grains number spike ${ }^{-1}$ and 100-grain weight at most cases under both water conditions. Kulshreshtha and Singh (2011) indicated that both additive and nonadditive gene action were found to be important in the inheritance of yield and yield attributes under normal as well as saline irrigation conditions. On the other hand, lqbal and Khan (2006) found that general combining ability effects were significant for spike length, number of spikelets per spike, number of grains per spike, spike density and grain yield. They added that the specific combining ability (SCA) variances were greater than general combining ability (GCA) variances, which showed the predominance of non-additive gene effects.

\section{Specific combining ability effects:}

Specific combining ability effects $\left(\hat{\mathrm{s}}_{\mathrm{i}}\right)$ of the parental combinations computed for all the studied measurements under normal and stress irrigation conditions are shown in Table (8). Seven and six crosses exhibited significant and negative (SCA) effects for days to heading in normal and stress irrigation conditions, respectively. Such results indicate that cross (Sahel $1 \times$ Sham 6) followed by cross (Gemmeiza $9 x$ Sham 6) could be considered as good crosses for developing line to early heading date.

Under normal irrigation conditions, six crosses exhibited negative and significant $\left(\hat{s}_{i j}\right)$ effects for days to physiological maturity, two crosses out of them didn't gave significant negative $\left(\hat{\mathrm{s}}_{\mathrm{i}}\right)$ effects for days to heading. Five crosses exhibited significant $\left(\hat{\mathrm{s}}_{\mathrm{i}}\right)$ effects for days to physiological maturity under water stress conditions, also two crosses out of them didn't gave significant $\left(\hat{s}_{\mathrm{ij}}\right)$ effects for days to heading. Such results confirm the viewpoint that it is not necessary that the genotype that have early heading date, have early maturity date also. Hence, maturity date could be reliable as a selection criterion for earliness than heading date. Earliness if found in wheat is favorable for escaping injuries by stress conditions (especially drought) and for intensive production.

Concerning plant height, among fifteen crosses, four crosses viz. (Sakha $93 \mathrm{x}$ Line 1), (Gemmeiza 9 x Line 1), (Misr $1 \times$ Sham 6) and (Misr $1 \times$ Line 1) showed significant positive $\left(\hat{\mathrm{s}}_{\mathrm{ij}}\right)$ effects at stress and normal irrigation conditions. The cross Misr 1 x Line 1 followed by Gemmeiza $9 \times$ Line 1 and Sakha $93 \times$ Line 1 showed highest significant positive $\left(\hat{\mathrm{S}}_{\mathrm{ij}}\right)$ under both irrigation conditions.

The most desirable $\left(\hat{\mathrm{s}}_{\mathrm{i}}\right)$ effects were recorded by the crosses (Sahel $1 \times$ Misr1), (Sakha 93 x Gemmeiza 9), (Gemmeiza 9 x Sham 6) and (Misr 1 x Line 1) under both normal and stress irrigation conditions for flag leaf area showing that these crosses appeared to be the best crosses for this trait. 
Table (8): Estimate of specific combining ability effects " $\hat{s}_{i j}$ " for the studied fifteen crosses under normal (N) and Stress irrigation (S) treatments for the traits studied.

\begin{tabular}{|c|c|c|c|c|c|c|c|c|c|c|}
\hline \multirow{2}{*}{ Crosses } & \multicolumn{2}{|c|}{ Days to heading } & \multicolumn{2}{|c|}{$\begin{array}{l}\text { Days to physio. } \\
\text { maturity }\end{array}$} & \multicolumn{2}{|c|}{ Plant height } & \multicolumn{2}{|c|}{ Flag leaf area } & \multicolumn{2}{|c|}{ Spike length } \\
\hline & $\mathrm{N}$ & $\mathrm{S}$ & $\mathrm{N}$ & $\mathrm{S}$ & $\mathrm{N}$ & S & $\mathrm{N}$ & $\mathrm{S}$ & $\mathrm{N}$ & S \\
\hline Sahel 1 x Sakha 93 & $-1.17^{\star \star}$ & -0.23 & $0.86^{\star *}$ & $1.08^{*}$ & -1.65 & $-2.60^{\star \star}$ & $2.89^{\star \star}$ & $6.35^{\star \star}$ & $0.66^{\star \star}$ & $0.38^{*}$ \\
\hline Sahel 1 x Gemmeiza 9 & 0.71 & -0.77 & $-0.93^{* *}$ & 0.79 & -0.36 & $5.29^{* *}$ & $-2.45^{\star \star}$ & $3.88^{* *}$ & $0.41^{*}$ & $0.38^{*}$ \\
\hline Sahel 1 x Misr 1 & $-1.25^{\star \star}$ & $-1.89^{\star *}$ & $-1.35^{\star \star}$ & -0.83 & -0.30 & -0.42 & $12.66^{\star *}$ & $12.26^{\star \star}$ & $0.71^{\star \star}$ & $0.53^{\star *}$ \\
\hline Sahel 1 x Sham 6 & $-4.63^{* *}$ & $-5.27^{\star \star}$ & $-2.39^{* *}$ & $-2.71^{\star *}$ & $-3.73^{\star *}$ & $2.10^{\star \star}$ & $-2.78^{\star \star}$ & $9.23^{\star *}$ & -0.16 & $0.88^{\star *}$ \\
\hline Sahel $1 \times$ Line 1 & -0.67 & -0.39 & -0.18 & $-1.50^{\star *}$ & 1.01 & $2.74^{\star *}$ & $-4.36^{\star *}$ & $-15.88^{\star *}$ & $-0.37^{*}$ & -0.14 \\
\hline Sakha 93 x Gemmeiza 9 & $-3.29^{* *}$ & $-1.60^{\star *}$ & $-1.39^{* *}$ & 0.63 & 1.39 & $2.30^{* *}$ & $6.81^{* *}$ & $10.62^{* *}$ & -0.07 & $0.45^{\star}$ \\
\hline Sakha 93 x Misr 1 & 0.42 & $0.94^{*}$ & $1.53^{\star \star}$ & $-2.33^{* *}$ & $2.23^{*}$ & 0.77 & $3.48^{\star *}$ & -1.34 & -0.16 & -0.20 \\
\hline Sakha 93 x Sham 6 & -0.29 & $-1.77^{\star \star}$ & $-1.18^{* *}$ & 0.46 & 0.01 & $2.54^{\star *}$ & $-3.79^{\star *}$ & -1.38 & 0.03 & 0.17 \\
\hline Sakha $93 \times$ Line 1 & 0.67 & 0.11 & 0.03 & $2.33^{* *}$ & $3.80^{\star *}$ & $3.70^{\star \star}$ & $-1.83^{\star \star}$ & $-2.25^{\star}$ & 0.16 & $0.36^{*}$ \\
\hline Gemmeiza 9 x Misr 1 & -0.71 & 0.07 & $-0.60^{*}$ & 0.37 & 1.80 & $-2.09^{\star *}$ & $-2.84^{\star *}$ & $-2.99^{* *}$ & $-0.36^{*}$ & $-0.47^{*}$ \\
\hline Gemmeiza 9 x Sham 6 & $-3.42^{\star *}$ & $-2.64^{\star \star}$ & $1.03^{\star *}$ & $-2.17^{\star \star}$ & $1.94^{*}$ & -1.17 & $10.07^{\star *}$ & $5.64^{\star \star}$ & 0.15 & -0.19 \\
\hline Gemmeiza $9 \times$ Line 1 & $0.88^{*}$ & $1.23^{\star \star}$ & $0.90^{\star *}$ & -0.96 & $3.45^{\star \star}$ & $4.01^{\star \star}$ & $-5.67^{\star \star}$ & $2.21^{*}$ & 0.10 & 0.05 \\
\hline Misr 1 x Sham 6 & $-1.04^{*}$ & $-2.10^{\star \star}$ & -0.05 & $-1.13^{*}$ & $2.13^{*}$ & $3.05^{\star *}$ & $-9.68^{\star \star}$ & $2.03^{*}$ & 0.03 & 0.33 \\
\hline Misr 1 x Line 1 & 0.58 & $1.11^{*}$ & 0.49 & -0.92 & $4.28^{\star \star}$ & $4.48^{\star \star}$ & $6.39^{\star *}$ & $9.85^{\star \star}$ & $0.51^{\star *}$ & $0.74^{\star \star}$ \\
\hline Sham $6 \times$ Line 1 & $-1.13^{*}$ & -0.60 & -0.55 & $1.54^{\star *}$ & $3.59^{\star *}$ & $-2.49^{\star \star}$ & $5.90^{\star *}$ & -0.17 & $0.78^{\star *}$ & $-1.34^{\star *}$ \\
\hline $\operatorname{LSD}\left(S_{\mathrm{ij}}\right) 0.05$ & 0.85 & 0.89 & 0.59 & 1.05 & 1.82 & 1.54 & 1.14 & 1.84 & 0.35 & 0.36 \\
\hline $\operatorname{LSD}\left(S_{i j}\right) 0.01$ & 1.14 & 1.18 & 0.79 & 1.40 & 2.43 & 2.06 & 1.52 & 2.47 & 0.47 & 0.48 \\
\hline $\operatorname{LSD}\left(S_{i i i}\right) 0.05$ & 0.70 & 0.73 & 0.49 & 0.87 & 1.50 & 1.27 & 0.94 & 1.52 & 0.29 & 0.30 \\
\hline $\operatorname{LSD}\left(\mathrm{S}_{\mathrm{ii}}\right) 0.01$ & 0.94 & 0.98 & 0.65 & 1.16 & 2.01 & 1.70 & 1.26 & 2.04 & 0.39 & 0.40 \\
\hline $\operatorname{LSD}\left(\mathrm{S}_{\mathrm{ii}} \mathrm{S}_{\mathrm{jj}}\right) 0.05$ & 0.96 & 1.00 & 0.67 & 1.18 & 2.05 & 1.74 & 1.28 & 2.08 & 0.40 & 0.40 \\
\hline $\operatorname{LSD}\left(S_{\mathrm{ii}} S_{\mathrm{jj}}\right) 0.01$ & 1.29 & 1.34 & 0.89 & 1.58 & 2.74 & 2.33 & 1.72 & 2.78 & 0.53 & 0.54 \\
\hline $\operatorname{LSD}\left(S_{i j}-S_{i k}\right) 0.05$ & 1.27 & 1.32 & 0.88 & 1.56 & 2.71 & 2.30 & 1.70 & 2.75 & 0.52 & 0.53 \\
\hline $\operatorname{LSD}\left(S_{i j} S_{i k}\right) 0.01$ & 1.70 & 1.77 & 1.18 & 2.09 & 3.63 & 3.08 & 2.27 & 3.68 & 0.70 & 0.72 \\
\hline $\operatorname{LSD}\left(S_{i j}-S_{k l}\right) 0.05$ & 1.18 & 1.22 & 0.82 & 1.45 & 2.51 & 2.13 & 1.57 & 2.55 & 0.48 & 0.50 \\
\hline $\operatorname{LSD}\left(S_{i j} S_{k l}\right) 0.01$ & 1.58 & 1.64 & 1.09 & 1.94 & 3.36 & 2.85 & 2.10 & 3.41 & 0.65 & 0.66 \\
\hline
\end{tabular}


J. Adv. Agric. Res. (Fac. Agric. Saba Basha)

Table (8): Cont....

\begin{tabular}{|c|c|c|c|c|c|c|c|c|c|c|}
\hline \multirow{2}{*}{ Crosses } & \multicolumn{2}{|c|}{$\begin{array}{c}\text { Number of } \\
\text { spikelets/spike }\end{array}$} & \multicolumn{2}{|c|}{$\begin{array}{c}\text { Number of } \\
\text { kernels/spike }\end{array}$} & \multicolumn{2}{|c|}{$\begin{array}{c}\text { Number of } \\
\text { spikes/plant }\end{array}$} & \multicolumn{2}{|c|}{$\begin{array}{c}1000 \text { kernel } \\
\text { weight }\end{array}$} & \multicolumn{2}{|c|}{ Grain yield/plant } \\
\hline & $\mathrm{N}$ & S & $\mathrm{N}$ & S & $\mathrm{N}$ & $\mathrm{S}$ & $\mathrm{N}$ & S & $\mathrm{N}$ & S \\
\hline Sahel 1 x Sakha 93 & $0.52^{* *}$ & 0.40 & 1.66 & 1.08 & $3.32^{\star \star}$ & 1.06 & $1.59^{\star *}$ & -0.06 & $5.67^{\star \star}$ & 1.23 \\
\hline Sahel 1 x Gemmeiza 9 & $0.72^{\star *}$ & 0.22 & -0.05 & -2.31 & $1.34^{*}$ & $1.48^{*}$ & $1.64^{\star *}$ & $0.93^{* *}$ & $9.15^{\star *}$ & 1.89 \\
\hline Sahel $1 \times$ Misr 1 & $-0.51^{\star *}$ & 0.39 & $-2.80^{* *}$ & $-5.83^{\star *}$ & 0.64 & $1.78^{* \star}$ & $2.62^{\star \star}$ & $2.91^{\star *}$ & 1.07 & $7.19^{\star *}$ \\
\hline Sahel $1 \times$ Sham 6 & $-0.68^{* *}$ & -0.28 & $-6.59^{* *}$ & $2.48^{*}$ & 0.90 & $2.27^{* *}$ & $1.24^{*}$ & $1.71^{* *}$ & $4.51^{* *}$ & $6.43^{\star *}$ \\
\hline Sahel $1 \times$ Line 1 & 0.15 & $0.70^{\star *}$ & $7.78^{\star *}$ & $10.05^{\star *}$ & -0.49 & -0.69 & 0.05 & 0.62 & $7.88^{\star *}$ & $6.63^{\star \star}$ \\
\hline Sakha 93 x Gemmeiza 9 & -0.17 & $0.53^{*}$ & $5.84^{* *}$ & $5.46^{\star \star}$ & 0.81 & $2.55^{\star *}$ & 0.04 & $2.34^{\star *}$ & $8.08^{* \star}$ & $10.59^{* *}$ \\
\hline Sakha $93 \times$ Misr 1 & 0.14 & $-0.62^{\star *}$ & $-3.84^{\star *}$ & $-6.59^{* *}$ & $5.25^{\star \star}$ & -0.40 & $2.72^{\star \star}$ & -0.43 & 3.18 & 0.37 \\
\hline Sakha 93 x Sham 6 & 0.11 & 0.15 & $-2.57^{\star}$ & -0.99 & $-2.06^{\star *}$ & -0.84 & $1.24^{*}$ & $1.32^{* \star}$ & $-5.57^{* *}$ & -1.06 \\
\hline Sakha 93 x Line 1 & $0.51^{* *}$ & 0.25 & $-2.25^{\star}$ & $-4.04^{\star *}$ & $1.50^{\star}$ & 2.89 & $3.05^{\star *}$ & $0.98^{* *}$ & $10.38^{* *}$ & $9.03^{\star \star}$ \\
\hline Gemmeiza 9 x Misr 1 & $-0.35^{\star}$ & -0.24 & $-5.20^{* *}$ & $-5.85^{\star *}$ & $-1.37^{*}$ & $1.29^{*}$ & $4.12^{\star \star}$ & $5.34^{\star *}$ & $-7.09^{* *}$ & 0.61 \\
\hline Gemmeiza 9 x Sham 6 & -0.24 & 0.27 & $-4.20^{* *}$ & 1.57 & 0.88 & $-1.50^{*}$ & $1.29^{*}$ & $-2.23^{\star *}$ & $3.70^{*}$ & -1.50 \\
\hline Gemmeiza 9 x Line 1 & -0.16 & $-0.50^{*}$ & $16.98^{* *}$ & $-9.60^{* *}$ & $2.80^{\star *}$ & -0.15 & $3.55^{\star *}$ & $3.67^{* *}$ & $7.43^{\star *}$ & $-4.38^{\star}$ \\
\hline Misr 1 x Sham 6 & $0.87^{\star *}$ & -0.13 & $-4.19^{* \star}$ & -1.59 & -0.39 & -0.07 & $1.21^{*}$ & $2.69^{* *}$ & 1.68 & 0.24 \\
\hline Misr $1 \times$ Line 1 & $0.51^{* *}$ & $0.64^{* *}$ & $8.14^{\star \star}$ & $11.95^{\star *}$ & $-1.79^{* *}$ & $-1.25^{\star}$ & $-1.47^{* *}$ & -0.21 & 2.68 & $5.78^{\star *}$ \\
\hline Sham $6 \times$ Line 1 & -0.05 & $-0.49^{*}$ & $3.85^{\star \star}$ & $-7.37^{\star \star}$ & 1.04 & -0.96 & $-1.96^{\star *}$ & 0.40 & $5.69^{* *}$ & $-5.43^{\star *}$ \\
\hline $\operatorname{LSD}\left(\mathrm{S}_{\mathrm{ij}}\right) 0.05$ & 0.32 & 0.44 & 2.00 & 2.41 & 1.17 & 1.16 & 1.05 & 0.70 & 3.27 & 3.35 \\
\hline $\operatorname{LSD}\left(\mathrm{S}_{\mathrm{ij}}\right) 0.01$ & 0.42 & 0.59 & 2.68 & 3.23 & 1.57 & 1.56 & 1.40 & 0.93 & 4.38 & 4.48 \\
\hline $\operatorname{LSD}\left(\mathrm{S}_{\mathrm{ii}}\right) 0.05$ & 0.26 & 0.36 & 1.65 & 1.99 & 0.97 & 0.96 & 0.87 & 0.58 & 2.70 & 2.77 \\
\hline $\operatorname{LSD}\left(\mathrm{S}_{\mathrm{ii}}\right) 0.01$ & 0.35 & 0.49 & 2.21 & 2.67 & 1.30 & 1.29 & 1.16 & 0.77 & 3.62 & 3.70 \\
\hline $\operatorname{LSD}\left(\mathrm{S}_{\mathrm{ii}}-\mathrm{S}_{\mathrm{jj}}\right) 0.05$ & 0.36 & 0.50 & 2.26 & 2.72 & 1.33 & 1.31 & 1.18 & 0.79 & 3.69 & 3.78 \\
\hline $\operatorname{LSD}\left(\mathrm{S}_{\mathrm{ii}}-\mathrm{S}_{\mathrm{jj}}\right) 0.01$ & 0.48 & 0.67 & 3.02 & 3.64 & 1.77 & 1.76 & 1.58 & 1.05 & 4.94 & 5.06 \\
\hline $\operatorname{LSD}\left(S_{i j} S_{i k}\right) 0.05$ & 0.47 & 0.66 & 2.99 & 3.60 & 1.75 & 1.74 & 1.57 & 1.04 & 4.89 & 5.00 \\
\hline $\operatorname{LSD}\left(\mathrm{S}_{\mathrm{ij}}-\mathrm{S}_{\mathrm{ik}}\right) 0.01$ & 0.63 & 0.88 & 4.00 & 4.82 & 2.35 & 2.32 & 2.09 & 1.39 & 6.54 & 6.69 \\
\hline $\operatorname{LSD}\left(S_{i j}-S_{k l}\right) 0.05$ & 0.44 & 0.61 & 2.77 & 3.34 & 1.62 & 1.61 & 1.45 & 0.96 & 4.52 & 4.63 \\
\hline $\operatorname{LSD}\left(\mathrm{S}_{\mathrm{ij}} \mathrm{S}_{\mathrm{kl}}\right) 0.01$ & 0.58 & 0.82 & 3.70 & 4.46 & 2.17 & 2.15 & 1.94 & 1.29 & 6.05 & 6.20 \\
\hline
\end{tabular}


Four crosses i.e. (Sahel $1 \times$ Sakha 93), (Sahel $1 \times$ Gemmeiza 9). (Sahel 1 x Misr 1) and (Misr $1 \times$ Line 1 ) had significantly positive $\left(\hat{S}_{i j}\right)$ effects for spike length under normal and water stress conditions. The cross (Sahel $1 \times$ Sham 6) expressed the highest $\left(\hat{s}_{\mathrm{ij}}\right)$ effects for spike length under water stress conditions.

Regarding number of spikelets/spike, five crosses out of the fifteen crosses exhibited significant desirable $\left(\hat{\mathrm{s}}_{\mathrm{ij}}\right)$ effects under normal irrigation conditions. Meanwhile, three out of the fifteen crosses exhibited significant desirable $\left(\hat{\mathrm{S}}_{\mathrm{ij}}\right)$ effects under stress conditions, only the cross (Misr $1 \times$ Line 1 ) exhibited significant desirable $\left(\hat{\mathrm{S}}_{\mathrm{ij}}\right)$ effects for this trait under both normal and stress irrigation conditions.

From the same Table (8), it could be noticed that the three crosses; (Sahel $1 \times$ Line 1), (Sakha $93 \times$ Gemmeiza 9) and (Misr $1 \times$ Line 1) under normal and water stress conditions are considered to be promising hybrids for improving number of kernels/spike, as they showed highly significant positive $\left(\hat{s}_{i j}\right)$ effects. The cross (Gemmeiza $9 \times$ Line 1 ) was the worst cross for this trait under both irrigation conditions.

Regarding number of spikes/plant, four crosses exhibited significant positive $\left(\hat{\mathrm{S}}_{\mathrm{ij}}\right)$ effects under normal irrigation conditions, the cross (Sakha $93 \times$ Misr 1) had the highest $\left(\hat{\mathrm{S}}_{\mathrm{ij}}\right)$ effect. Meanwhile, under water stress conditions five crosses exhibited significant positive $\left(\hat{\mathrm{s}}_{\mathrm{ij}}\right)$ effects, the cross (Sahel $1 \times$ Gemmeiza 9 ) was the only cross which expressed significant positive $\left(\hat{\mathrm{s}}_{\mathrm{ij}}\right)$ effects for number of spikes/plant under both normal and water stress conditions.

Eight crosses exhibited significant positive $\left(\hat{\mathrm{s}}_{\mathrm{ij}}\right)$ for 1000 -kernel weight under both normal and water stress conditions. The crosses (Gemmeiza $9 \times$ Misr 1) and (Gemmeiza $9 \times$ Line 1) could be considered as the best crosses for this trait.

Concerning grain yield/plant, nine parental combinations showed significant positive $\left(\hat{\mathrm{s}}_{\mathrm{ij}}\right)$ effects under normal irrigation conditions. Also six crosses showed desirable $\left(\hat{\mathrm{S}}_{\mathrm{ij}}\right)$ effects under water stress conditions. The two crosses (Sakha 93 x Gemmeiza 9) and (Sakha $93 \times$ Line 1) seemed to be the best combinations for this trait under both normal and stress conditions.

\section{CONCLUSION}

The results revealed that there was significant genotypic variation among the genotypes for the studied traits. The genotype Line $1\left(\mathrm{P}_{6}\right)$ could be used as donor parent for the improvement of days to heading, days to physiological maturity, flag leaf area, spike length, number of kernels/spike and 1000-kernel weight for normal and water stress conditions. The cultivar Misr $1\left(\mathrm{P}_{4}\right)$, showed potential for the improvement of grain yield/plant under both irrigation conditions, and grain yield/plant under water stress conditions. The crosses (Sakha $93 \times$ Gemmeiza 9) and (Sakha $93 \times$ Line 1) have potential for the improvement of number of spikes/plant and grain yield/plant for water stress conditions. The cross (Sahel $1 \times$ Misr 1 ) holds a promise for yielding better segregates with improving flag leaf area. 


\section{REFERENCES}

Abd Elnour, N. A. (2005). Genetical studies for yield and its components on drought and drought susceptibility index in wheat. Egypt. J. Agric. Res., 38(4): 1725-1740 .

Ahmadi, J., A.A. Zali, B.Y. Samadi, A. Talaie, M.R. Ghannadha and A. Saeidi (2003). A study of combining ability and gene effect in bread wheat under stress conditions by diallel method. Iranian J. Agric. Sci., 34(1):1-8.

Elisabeth, S., D.G.F. Evan, T. Mette, M.F Piers and J.D. Andrew (2009). Typologies of crop drought vulnerability: an empirical analysis of the socio-economic factors that influence the sensitivity and resilience to drought of three major food crops in China (1961-2001). Envi. Sci. and Policy, 12: 438-452.

Golparvar, R.A. (2013). Genetic control and combining ability of flag leaf area and relative water content traits of bread wheat cultivars under drought stress condition. Genetika, 45(2): 351-360.

Griffing, B. (1956). Concept of general combining ability in relation to diallel crossing system. Aust. J. Biol. Sci., 9: 463-493.

Hakim, M.A., M.G. Rasul, M. Salimuddin, S.A. Bagum and N.C.D. Barm (2007). Studies on combining ability in spring wheat (Triticum aestivum, L.). Int. J. Sustain. Agri. Tech., 3(5):87-92.

Iqbal, M. and A. A. Khan (2006). Analysis of combining ability for spike characteristics in wheat (Triticum aestivum L.). Internat. J. of Agric. \& Bio., 8 (5): 684-687.

Joshi, S.K., S.N. Sharma, D.L. Singhania and R.S. Sain (2004). Combining ability in the F1 and F2 generations of diallel cross in hexaploid wheat (Triticum aestivum L. em. Thel.). Hereditas, 141(2):115-121.

Khan, M.A., N. Ahmad, M. Akbar, A.U. Rehman and M.M. Iqbal (2007). Combining ability analysis in wheat. Pak. J. of Agric. Sci., 44(1): 1-5.

Kulshreshtha, N. and K.N. Singh. (2011). Combining ability studies in wheat (Triticum aestivum L.) for genetic improvement under salt stress. J. Wheat Res., 3(2): 22-26.

Mohammadi, H., M.K. Emami and A. Rezai (2007). Estimation of genetic parameters for wheat grain yield and its components using diallel crosses. J. Sci. \& Tech. Agric. \& Natur. Resour., 11(40): 157-165.

Pang, H., W. Li, H. Zhang, L. Wahg, Y. Yin; H. Yuan and Z. Wang (2010). Inheritance analysis of resistance starch contents in kernels of wheat. Yi Chuan, 32(2): 170-176.

Saeed, A., A.S. Khan and I.Khaliq (2010). Combining ability studies on yield related traits in wheat under normal and water stress conditions. Pak. J. of Agric. Sci., 47(4): 345-354.

Seboka, H., A. Ayana and H. Zelleke (2009). Combining ability analysis for bread wheat (Triticum aestivum L.). African J. Sci., 3(1):87-94.

Steel, R.G.D., J.K. Torrie and D.A. Dickey (1997). Principles and procedures of statistics. A biometrical approach (3 ${ }^{\text {rd }}$ Ed.). McGraw Hill Book Co. Inc. New York, USA; pp.352-356.

Sultan, M. S., A. H. Abd El-Latif, M. A. Abdel-Moneam and M. N. A. El-Hawary (2011). Genetic parameters for some yield and yield components characters in four crosses of bread wheat under two water regime treatments. J. Plant Production, Mansoura Univ., 2 (2): 351-366. 


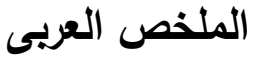

\section{القدرة على الإئتلاف لمحصول القمح ويعض مكوناته تحت الظروف العادية وظروف الإجهاد المائى}

\section{محمد نجيب البنا*، محمود عبد العزيز جمعه*، محمد عبدالجواد نصار *، حسن عبداللطيف عشوش***،

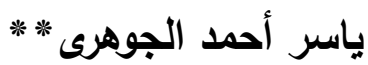 \\ * قسم الإنتاج النباتى (محاصيل) - كلية الزراعة سابا بانشا - جامعة الإسكندرية

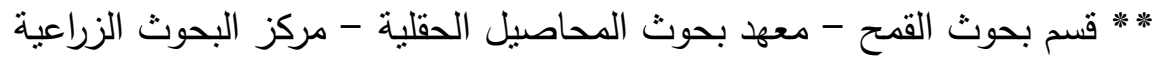

أجريت هذه الدراسة بمزرعة محطة البحوث الزراعية بإيتاى البارود خلال موسمي 2010/2009 و 2011/2010 وتهدف

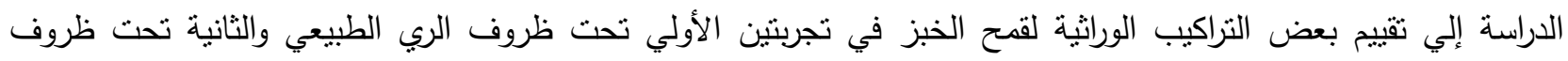

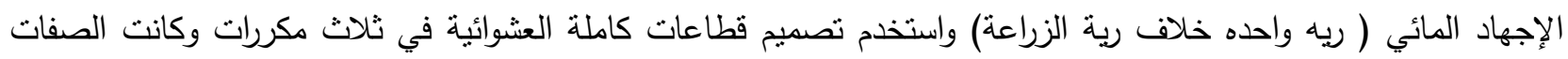

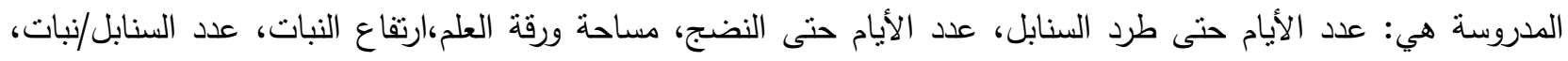

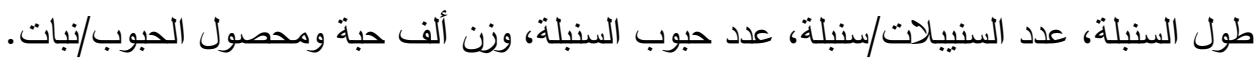

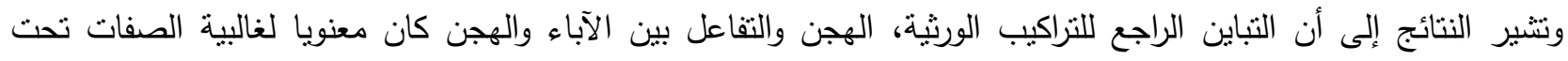

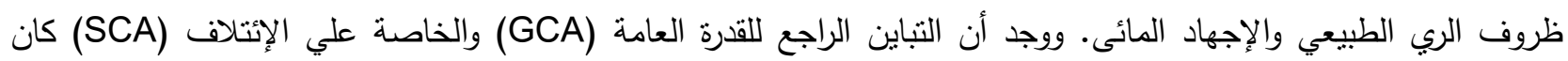

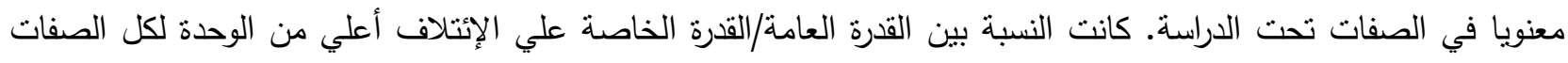

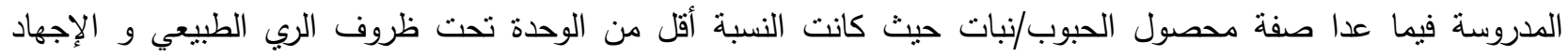

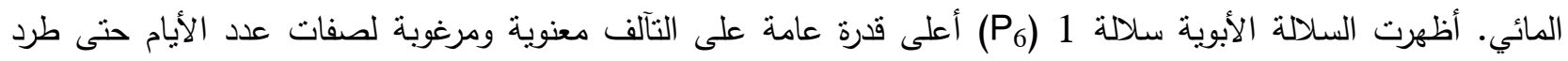
السنابل، عدد الأيام حتى النضج، مساحة ورقة العلم وطول السنبلة تحت ظروف الإجهاد المائى وصفتى عدد حبوب السنبلة و وزن ألف حبة تحت كل من ظروف الريام حنى الطن الطبيعي و ظروف الإجهاد المائي.

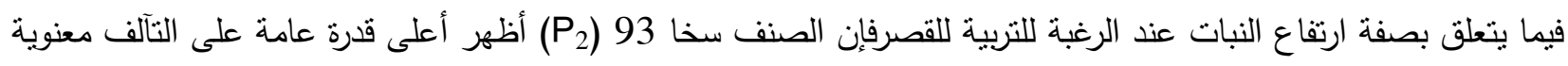

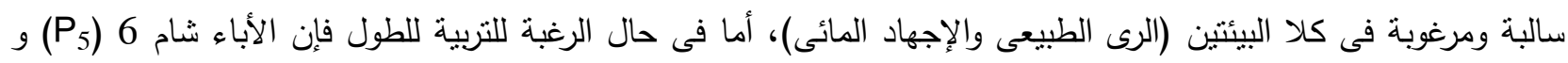

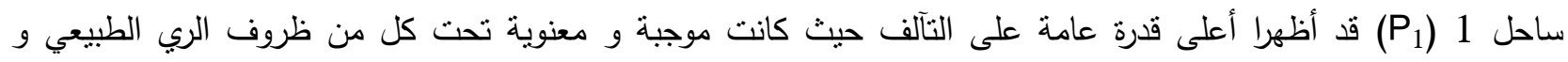

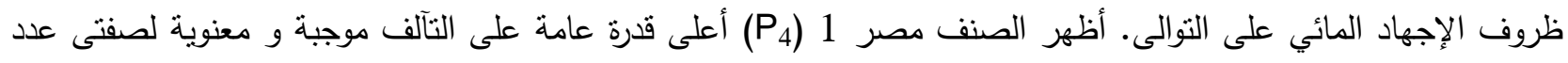

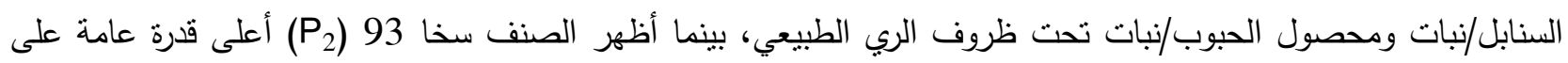

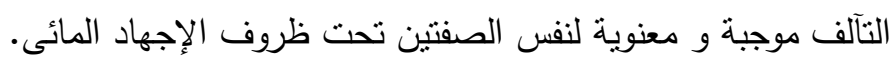

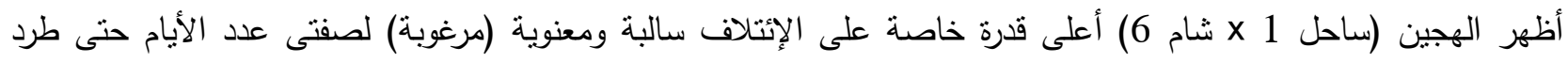

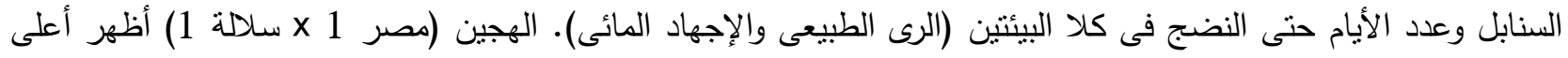




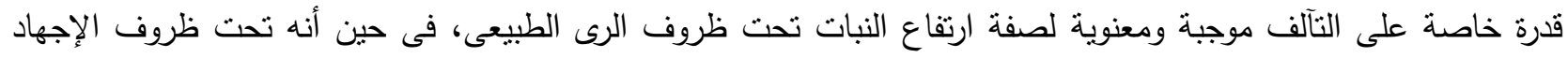

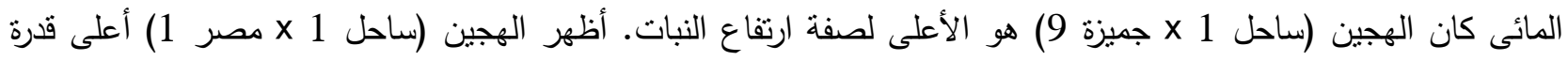

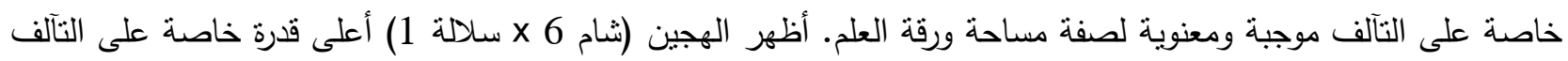

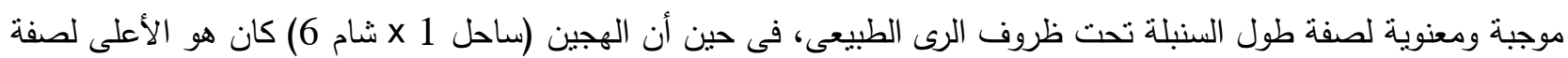

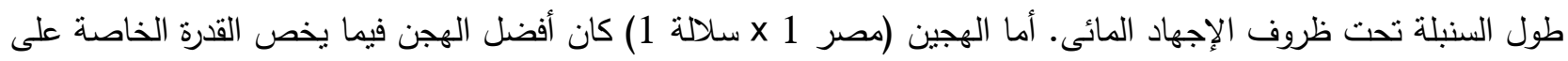

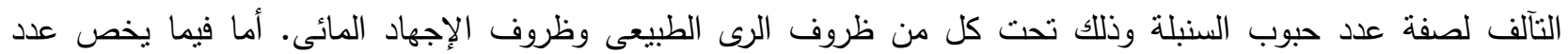

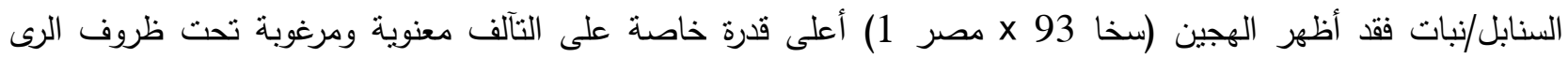
الطبيعي بينما تفوق الهجين (سخا 93 x سلالة 1) تحت ظروف الإجهاد المائى لصفة عدد السنابل/نبات.

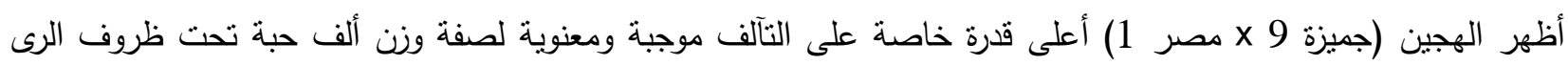
الطبيعى والإجهاد المائى. أظهر الهجين (سخا 93 x مصر 1) أعلى قدرة خاصة على التآلف موجبة ومعنوية لصفة عدد الطئ السنابل/نبات تحت ظروف الرى الطبيعى، فى حين أن الهجين (سخا 93 x جميزة 9) كان هو المتفوق لصفة وزن الأل الألف حبة

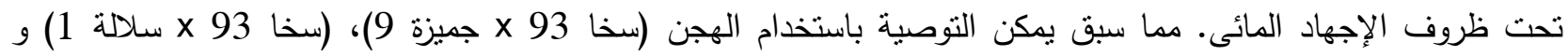

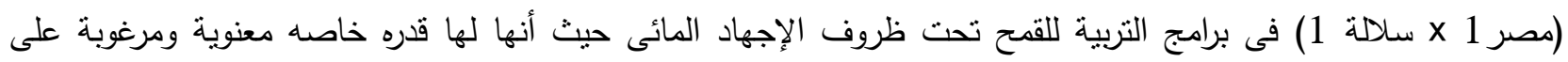

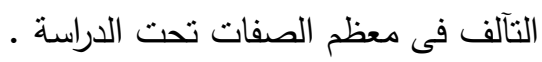

